\title{
Scalability conditions of the measuring points network in a production system using the RFID selective gate technology
}

\author{
Monika Łobaziewicz ${ }^{1, *}$ \\ ${ }^{1}$ DataConsult Sp. z o. o., 30-614 Kraków, Poland
}

\begin{abstract}
The aim of paper is to present results of the research work in development conducted to define the scalability conditions for the measuring points network at production cells in the resistant to the electromagnetic disturbances system based on the patented selective RFID gate technology. This is a conceptual paper that includes research results of the selective RFID gate and its place in a production system, a communication model between two measuring points in a complex production system that was the input to do further analysis required to define its scalability conditions. The proposed approach provides the following results: order and speed of data reading from measurement points, data transfer between measurement points, access to database, communication between the database and measurement points, data archiving, procedures of changing the measurement point configuration in the system, including error handling and network re-configuration. It is believed that both practitioners and researchers will benefit from it.
\end{abstract}

\section{Introduction}

An infrastructure of modern production systems consists of technologically advanced machines, devices and production lines that automate manufacturing processes. The reliability of communication between elements of the infrastructure functioning in a production area is one of the important problems to ensure the quality, stability, security of production and the right identification of resources used in the production processes [1-4]. Nowadays, manufacturing enterprises expect such communication systems that provide fast and accurate data flow in real-time [5-7], are resistant to the disturbances of machines and devices placed in the production area and collaborate smoothly with other outside systems.

The above premises supported by an analysis of market trends and the identification of problems during the implementation of IT systems for production and warehouse logistics were a source of inspiration for a research conducted by DataConsult Sp. z o. o. in $\mathrm{N}=104$ manufacturing companies. The research has shown that one of many technological barriers in the communication among elements of a manufacturing infrastructure is the electromagnetic disturbances caused by waves emitted by the machinery and production equipment, causing serious interruptions in the functioning of production lines. It turns out that the creation of a comprehensive measuring points system using a selective RFID gate technology meets the identified problems of enterprises because it allows to modify and develop production lines with new industrial automation components without the need to stop production or shorten the time required to make necessary changes to a minimum.

The aim of this article is to present the results of development work conducted to define the scalability conditions for the measuring points network at production cells in the resistant to electromagnetic disturbances system based on the selective RFID gate technology. An industrial research has been the input for the development work that included the communication model between two measuring points located at the production cells using the selective RFID gate. The model was based on the assumption of simultaneous independence of the communication from the configuration of industrial automation components, which enables a high level of security for collection, processing and sharing of data. This model is the result of a R\&D project called "Resistant to electromagnetic disturbances prototype of the measuring points system at production cells based on the selective RFID gate technology" made by DataConsult Sp. z o. o. in 2011 2014.

The article consists of five parts. The first part is an introduction to the issues discussed in the article. The second part presents the concept of a selective RFID gate and its role in the communication system among measuring points located in production cells. In the third part the communication model between two measuring points using the selective RFID gate was presented, while the fourth part presents determinants of its scalability in a complex production system. The last part is a summary of the issues discussed in the article.

\footnotetext{
* Corresponding author: ml@un.pl
} 


\section{Selective RFID gate}

\subsection{Results of the research using EMC absorption materials to eliminate disturbances in radio communication systems}

The state of the art [8-12], [17-19], shows that despite of its popularity RFID technology all over the world continues to develop and provides new opportunities for Automatic Data Capture (ADC) in various industries. The aim of using RFID is to share data encoded in tags and reading them in time and place required by adopted solutions of the system [13, 14]. The typical RFID activity is based on a set of antennas connected to a controller and embedded on a company specific structure called the RFID gate $[15,16]$. It turns out that currently most of RFID gates used in enterprises operate in a non-selective way, meaning that not only the tags passing through the light of the gate, but also those next to, and even behind, can be read in the identification process. This gives an incorrect identification of objects moving along a production line.

Therefore, at the stage of industrial research carried out by DataConsult Sp. z o. o. in the cooperation with Institute of Logistics and Warehousing in Poznan, the approach as in the electromagnetic compatibility research was used, absorbing radio waves and eliminating unwanted phenomena such as collapse and reflection of waves. It has been verified whether and when it is possible to screen undesired back-light RFID antennas using EMC absorbers which is an important limitation for the correct objects' identification during production processes. The research was made by measuring the intensity of electromagnetic field in the fixed area behind the gate. For this purpose, a grid of measuring points was used to form a square of $1.2 \mathrm{~m}$ at the height of the RFID antenna (Fig. 1).

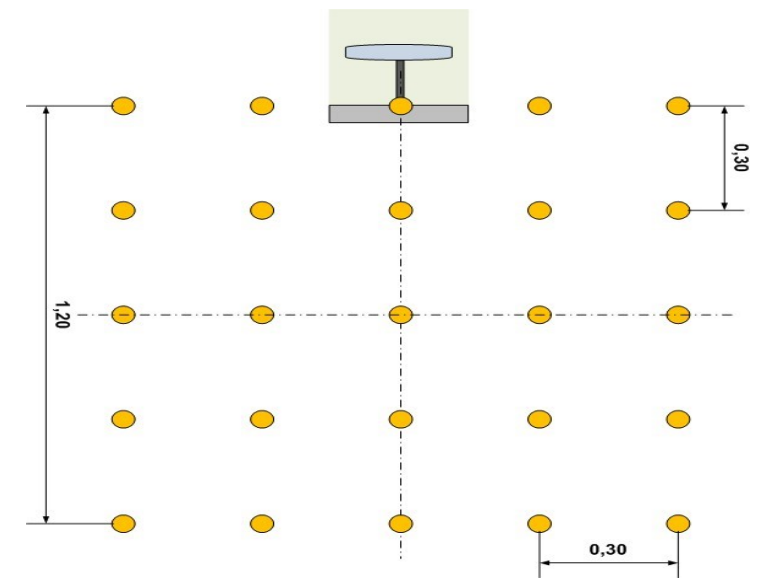

Fig. 1. A grid of measuring points for the measurement of field intensity situated out of the RFID gate.

The results have shown that the use of tapered absorbers significantly reduces the field intensity generated by the antenna out the RFID gate and thus eliminates unwanted tag readings [20].

\subsection{Construction of the selective RFID gate}

The research results presented above became the input to design the selective RFID gate (Fig. 2). It is the pioneering use of this device in the manufacturing industry and the second in the world [21]. In 2016 the Patent Office of the Republic of Poland has issued the patent PL 222069 B1 for the selective RFID gate [22].

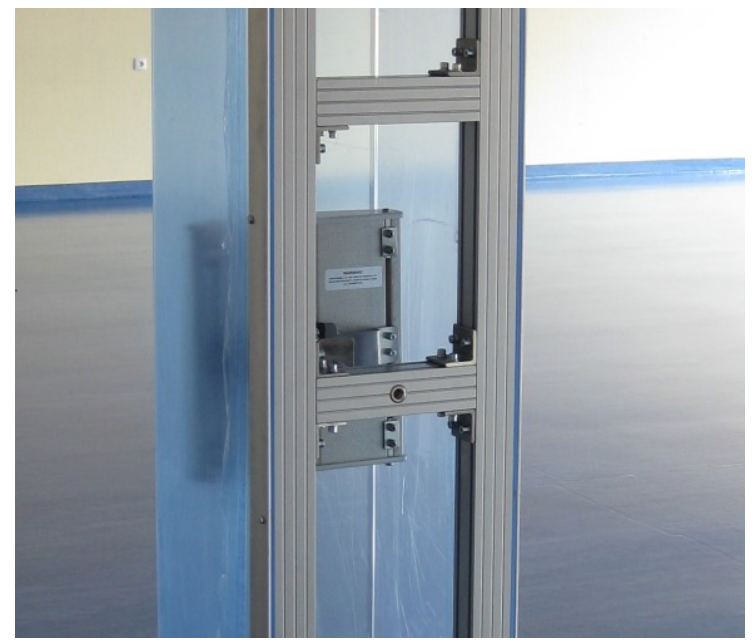

Fig.2. Element of the selective RFID gate.

Actually, most of the RFID gates are based on metal constructions that can interact with radio waves used in RFID systems as information carriers. As a result, the RFID gate is not selective, meaning that it can read data from tags placed not only at its "light area" but also outside it. In addition, the typical RFID gates do not allow creating the area to be readable as a result of the characteristics of antennas used and often require the specific organization of the gate environment so that there are no undesirable markers in the range of the readings [23].

The selective RFID gate reads information from tags passing only through the gate's light that creates a space inside the device. As a result, the selective RFID gate is highly resistant to electromagnetic disturbances, allowing for densification of measuring points in production without increasing the risk of reading redundant tags. In the next part of the article its application in the communication among measuring points located on the production line is presented.

\section{A communication model between two measuring points in a complex production system}

This part presents two aspects. The first one concerns the ways of communication among measuring points that have been identified in industrial production systems. The results of the analysis were employed to develop a communication model between two measuring points in a complex production system. 


\subsection{Communication among measuring points in a production system}

The industrial research concluded that in practice there are several variants of communication among measuring points in a production system.

\subsubsection{Direct communication between RFID tags readers and Ethernet}

One of the communication ways in a production area is the direct communication connecting RFID tag readers to Ethernet using standard LAN and WLAN ports. In consequence, it allows the configuration of communication among measuring points based on the IEEE LAN/ WLAN communication standard. Thus, it imposes a choice of communication interface based on $\mathrm{TCP} /$ IP protocols. On the one hand, this solution makes it easy to implement it into the production system and on the other hand, a data collection for reporting and controlling requires additional devices. A disadvantage of this approach is the lack of certainty that the communication between the points is in the correct order according to the logic of a manufacturing process.

\subsubsection{Measuring points communication based on database}

The second approach is communication between measuring points and a database. The Ethernet and a built-in internal data communication protocol with RFID tags allow a reader to connect to an external data server. In this approach each of RFID readers may have an assigned IP address during a system configuration. It is also necessary to define the appropriate order of asking the measuring points about database server services. Reading RFID tag with a unique TID/EPC code by a specific reader places a resource at a specific location. Then, the information about the registration of transition of the marked resource is transferred to the database which can be saved for reporting purposes or for further processing. This solution gives good conditions for the scalability. Data collection by the database enables to monitor of all tagged assets, particularly "lost" and "abandoned" which can be identified by an operator having a portable RFID tag reader. The acquisition of data beyond RFID readers minimizes the effects of a device or software failure and the related consequences, e.g. the loss of already accumulated data.

\subsubsection{Measuring points communication based on RFID tags with the limited access to Ethernet}

The third approach of communication between measuring points is the use of RFID tags when the communication is limited by the access to Ethernet. The research concluded that RFID readers mostly work under Windows CE and have a flash memory. Each of measuring points equipped with the RFID reader independently reads a tag and directs it to a corresponding process on the basis of information contained in its memory. This is a secure solution but limits the ability to supervise measurement processes only to those measuring points that can be connected to Ethernet or where surveillance procedures have been defined. Although it is possible to include additional sums recorded in tags that inform about the quantity or a number of objects passing through the measuring points, however, due to the limited capacity of tags' memory, it can turn out to be inadequate. This solution makes each measuring point operate independently and requires the installation of up-to-date software that will work with all the other measuring points. This configuration requires manual and simultaneous software updating of the measuring points, which may cause the increase of system maintenance costs and require technical breaks.

The analysis leads to the conclusion that the optimal variant of communication among the measuring points is to use a database. There are new functionalities added to a communication system and additional measuring points are connected. It monitors the flow of objects and collects real time data. The communication based on the database enables a quick identification of technical problems that can appear during the work of measuring points.

\subsection{The concept of a communication model between two measuring points in a complex production system}

The analysis results of available communication approaches between measuring points have become the basis for creating a communication model between them based on the SQL database server (Fig.3).

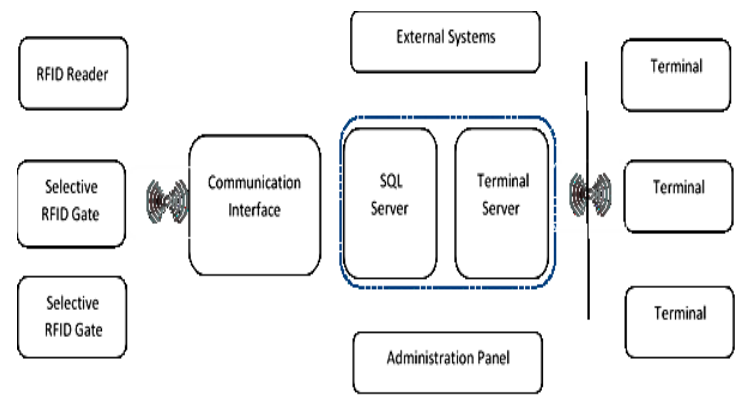

Fig. 3. The communication model between two measuring points with use of the selective RFID gate.

The model's architecture [24] requires a software installation in the measuring points linked to a database server by the dedicated service. The measuring points $\log$ to the terminal server that sends information generated from tags to the database. Simultaneously, parameters such as the time of tagging and the gate's IP are stored in the database.

In communication between two measuring points it is important to define what data will be transmitted between them. It enables the defining of measuring points behavior that is determined by the production line automation system, e.g. the objects flow with the defined 
EPC code and the wrong weight may result the light or sound alarm on the line.

In a communication model between two measuring points many system components can be used, especially processes and technologies.

As far as processes are concerned, the recording of material in a production cell can be treated as an individual process or as a command generating new actions, the realization of which will be determined by a tagged asset passing through the next measuring point.

With reference to technology, a production order is when the input measuring point records objects through RFID tags before they come to a production hall and the output measuring point automatically registers and controls products leaving a production hall.

The research show that Universal Data Exchange Interface (UDEI) is the optimal standard for the communication between measuring points which is responsible for exchanging information with any external system, e.g. ERP. Using dedicated transition tables information is exchanged between the measuring points software and the system database tables. Data processing configurations are implemented in the database enabling the implementation of pre-defined measuring points actions.

To control and configure the system it has a dedicated communication interface that includes: Services, Cycle Tasks, Tasks, Templates, Document Types, Warehouses. The characteristics of configurations are available in ERP system. It has been assumed that the communication interface between two measuring points requires validation tests. The parameters of communication interface should be tested during the analysis and at the implementation stage. An important element required for the implementation of communication interface is to provide an adequate way of information presentation aggregated from the measuring points.

During the research work in development the communication model between the measuring points at the production line using the selective RFID gate was tested in conditions similar to the reality. The tests provided the following results: the correct identification of resources flowing through the RFID gate; the system has shown significantly improved resistance to electromagnetic disturbances; there are no limitations with the growing number of measuring points or distance shortening between production lines. The risk of reading an object from the next line does not exist; the system automatically records information in RFID tag; the easy integration with external IT systems and industrial automation devices; the high flexibility to adapt the system to the existing enterprise production system.

\section{Scalability determinants of a communication model between measuring points in a complex production system}

At the research work in development the factors that determine the scalability of the communication model between the two measurement points included: order and speed of data reading from each measurement points, data transfer between measurement points, access to database, communication between the database and measurement points, data archiving, procedures of changing the measurement point configuration in the system, including error handling and network reconfiguration.

\subsection{Measuring points synchronization}

\subsubsection{Order and speed of data reading from measuring points}

The development work has shown that during a production process it is possible to define behaviour of measuring points depending on the recorded data. The order in which RFID tags are scanned is stored in system database tables, which is determined by the production process. The appearance of a non-registered element in the RFID tag at the measuring point triggers an action of industrial automation elements at the selective RFID gate. The use of triggers activates procedures for updating data in database tables when the problem occurs at a measuring point. Therefore, the chronology of RFID tags collected from the measuring points is controlled. The communication defined in this way causes the measuring points to have an active process of adding records in the transition tables. Because the RFID readers are equipped with Windows $\mathrm{CE}$ operating system, it is necessary to check if the process has started properly and works in all the measuring points. For this purpose, the system must register login and logout information of all the measuring points in the system database.

In a communication model between two measuring points the Universal Data Exchange Interface (UDEI) standard was used. As far as scalability of a system is concerned, it allows the setting of the time of cycle tasks and the defining of the time required for messaging between the database and each of the measuring point. In a production system this solution enables the communication speed between the measuring points to be adjusted to their configuration. It requires setting the maximum time necessary for communication between measuring points and the system database. These data should be established during the pre-implementation analysis and based on the identified production processes in the enterprise.

\subsubsection{Data transmission principles between the measuring points}

An indirect communication approach in which a database with indirect tables is used is also accepted in a communication model between two measuring points. In practice, this requires defining the appropriate behaviour of database triggers activating data transfer services. For this purpose, a detailed analysis of production processes creating the manufacturing system is necessary, taking into account the role of measuring 
points in the production line. Scaling of the communication system requires determining the level of significance of transmitted data among measuring points that can be divided into two groups:

- Critical data transmitted among measuring points with the highest priority and the shortest time available for the system. Such data may include, for example, events that threaten the safety of people working on the production line, resulting in unplanned downtime. For this type of data, it is assumed that the database triggers make active service that communicates with the measuring points to forward information about taking the action. Depending on the security level defined during the system implementation, it can be configured as a service whose aim is to perform tasks of the critical communication among measuring points;

- Non-critical data providing information. They can be communicated by services in cycle tasks that make work time flexible to ensure optimal company performance under the stable production conditions with the optimal network infrastructure load and database needed to ensure the critical communication.

\subsection{Database management}

The database management process consists of an access to database, the communication efficiency between the database and measuring points and data archiving.

\subsubsection{Multiple access to the database}

During communication between measuring points via database, the process is as follows: reading data from the measuring point $\rightarrow$ saving data in database tables $\rightarrow$ processing data $\rightarrow$ reading data from database tables $\rightarrow$ sending data to the measuring point. In this approach, reading and sending data from/to the measuring point is fully controlled. Problems can arise while saving, processing and reading data in database tables.

It is assumed that database tables are locked only during data saving while reading data from tables. The access to them for the other tasks is possible via the command "no lock". This significantly shortens the access time to data stored in the database and facilitates quick and repeated access to the data.

It turned out that it is a significant problem to save data in database tables. During this action, only one task "point-to-point" communication is available in the table when data is saved or updated. Database server with the appropriate parameters can significantly reduce the time of the table lock. During the research work in development, it was decided that the database server should provide a minimum $200 \mathrm{MB}$ of RAM for each measuring point.

\subsubsection{Communication efficiency between database and measuring points}

When defining the scaling conditions of the designed system, it turned out that the communication efficiency between the databases and measuring points is time- dependent. In the basic configuration, the communication process is carried out as a single thread and tasks are queued. In order to improve the access time to the database, it is good to use multithreading. Then, the maximum benefit is in inverse proportion to the maximum number of configured threads available for the communication service. Two basic limitations have been identified in this case. First of all, when data is saved or updated the table in which the task is going on remains locked for others. As a result, the communication time between measuring points is reduced. Secondly, the use of multithreading means that tasks coming later through a communication service from a measuring point can be done in a new thread earlier. If there is no control over the order of executed tasks in every measuring point, introducing multithreading can result in the information about the lack of resources or generate extra actions by measuring points "seeking" production resources. The multithreaded service communication requires task modifications to control the communication time of preceding measuring points in the production hierarchy more accurately.

\subsubsection{Data archiving}

During the research work in development the following mechanisms to control the historical data collection have been implemented:

- Historical tables where data is transferred after a production process is finished. Data is not used by the database anymore for communication among the measuring points but can be used for accounting or reporting;

- Data archiving - the time after which the collected data is not used by users and should be exported to text files should be defined. Such a solution affects the security level of the system.

In communication among multiple measuring points the order of data flow is an important issue for the correct data exchange. Preceding measuring points in the production cycle should have access to the database in order to update data needed for the next points. It means saving all historical data collected by the measuring points identified as critical in the production process with the time of their reading at the measuring point in the database.

\subsection{Procedures of configuration changes in the measuring points network}

The next issue related to the scalability of communication among multiple measurement points, was failure handling and network reconfiguration. It turned out that as the number of measuring points increased, the configuration and communication complexity of the measurement and communication system exponentially increased. This can cause a significant increase of time necessary to remove any system failures or to plan longer and longer technical breaks to reconfigure measuring points. Thanks to Ethernet in the interface design, each measuring point 
has an assigned IP address, uniquely defining the location of the point in the production process. Assigning a unique MAC address for each measuring point makes data collection from the measuring equipment installed in it possible. This solution changes topology and reconfigures the measuring network by moving measuring points, if necessary.

\subsubsection{Handling of failure in the measuring points}

In order to handle failures in the communication between the measuring points correctly, the following parameters are required:

- Time to establish communication between measuring point and database, and

- Quantity and quality of data.

If during the communication between a measuring point and database any of the defined parameters in the system is not sent or is not in the right format, one of the following messages will be generated: critical (missing data required to continue a production process), or warning (information about the lack of data not directly affecting a production process).

Based on the defined failure information, the database trigger activates a failure procedure. It may contain different scenarios, such as devices restarting in a measuring point and attempting to re-establish communication, restarting the communication task, starting emergency procedures in other points, stopping the production process.

The research work in development concluded that in case of failure, the communication system gives an opportunity for analysis using historical data and data from measuring points located logically near to the failure point. It makes a remote pre-analysis of the event possible

\subsubsection{Network reconfiguration}

In the research work in development on the scalability of multiple measuring points, it was also required to define circumstances of system development and its modification. It has been pointed out that the measuring points system may be changed in three ways.

\subsubsection{Changes of measuring points topography}

The communication system is designed to independently identify measuring points, thus topography changes may refer to repositioning of the measuring points within the same topology. Each modification requires changing the corresponding IP or MAC address in the database and in the communication and production processes defined in the database. These works do not take much time because they rely on activating the script in the system database. The problem that can occur during a configuration change may be due to the limited ability to validate changes before they start.

\subsubsection{Measuring points system development}

The system topology development is accomplished by adding new IP/MAC address to the system database. They will remain inactive until it has configured the procedures and operations that will be used. The database development allows a new production system based on new measuring points to be tested.

\subsubsection{Reduction of network of measuring points}

The research work in development has shown that in order to reduce the number of measuring points the corresponding IP/ MAC address from the database must be removed and all processes and procedures using the deleted points are modified. The network reduction should be preceded by a detailed analysis of the network topology of measuring points in order to identify early production lines that could lead to unfinished final products. The problem that may occur during the network redundancy will be due to the limited ability to validate changes before they begin, which is similar to changing the topography of measuring points.

\section{Conclusion}

The input for the research work in development presented in the paper was the output of the previous research stages focusing on the design of selective RFID gate and then a communication model between two measuring points in a complex production system.

The main factors that determine the scalability of the communication between the measurement points network are as follows: order and speed of data reading from each measurement points, data transfer between measurement points, access to database and communication between the database and measurement points, procedures of changing the measurement point configuration in the system, including error handling and network re-configuration.

In case of order and speed of data reading from measurement points, the research shows that it is required to check at first if the process of reading RFID tags has started correctly and works in each of the points. Therefore, the system must register login and logout of all the measuring points in the database. The communication requires all the measuring points to have an active process of adding records in the transition tables. In addition, one should set the maximum time required for communication between measuring points and the system database.

The significance of transmitted data, critical or noncritical, between measuring points needs to be determined to make scaling of the communication system successful. It depends on the structure of manufacturing processes in a production system.

The next aspect is the access to database. The preceding measuring point in the production cycle should have access to the database in order to update data needed for the next point. The server should provide a minimum $200 \mathrm{MB}$ of RAM for each measuring point. 
In order to improve the access time to the database, it would be good to use multithreading, even though it has some limitations. One of them is that tasks coming later through a communication service from a measuring point can be done in a new thread earlier. As a result, it can provide information about the lack of resources or generating additional actions by measuring points "seeking" production resources. It means that the multithreaded service requires task modifications to control the communication time of previous measuring points in the production hierarchy more accurately.

The research shows that as the number of measuring points grows up, the configuration and communication complexity of the system exponentially increases, and the probability of system errors is higher. Thus, the system is able to make analysis using historical data and data from measuring points located logically near to the failure point. It makes a remote pre-analysis of the event possible.

Any modifications of the measuring points network are related with the system topology. Adding or deleting measuring points requires changing the corresponding IP or MAC address in the database and in the communication and production processes defined in the database. This should be preceded by a detailed analysis of the network topology of measuring points in order to avoid serious problems at production lines.

Summing up, this work introduces the solution based on the theoretical research and some practical ideas that were designed by DataConsult in the collaboration with the Institute of Logistics and Warehouse. The research owes its success to the selective RFID gate that was patented in 2016. The validation of measuring points network with use of the selective RFID gate in a few production systems confirmed its effectiveness in real manufacturing conditions. Therefore, the results of the research work in development presented in the article can be useful for industry practitioners, IT experts, and researchers who might need to work together to build a smarter solution in the future.

\section{References}

1. D.C.Wimalachandra, B. Frank, T. Enkawa, Int J Ind Eng-Theory, 21, 348-359 (2014)

2. D.A.Perry, B. Olson, P. Blessner, T.D. Blackburn, Systems Eng, 19, 133-145 (2016)

3. C.K.H Lee, K.L. Choy, K.M.Y.Law, G.T.S Ho, J
Manuf Syst, 3, 412-422 (2014)

4. M. Ghobakhloo, S.H. Tang, S.H., Inform Technol Dev, 21, 573-600 (2015)

5. J.Smart, A. Calinescu, L.H. Huatuco, Int J Prod Res, 51, 362-379 (2013)

6. J. Santos, M.P.Garcia, M. Arcelus, E. Viles, J. Uranga, Int J Comput Integ M, 24, 338-351 (2011)

7. C.Z. Zulkifli, H.N. Hassan, W. Ismail,S.N. Semunab, Acta Phys Pol A, 128, B86-B89 (2015)

8. E. Dovere, S. Cavalieri, S., S.Ierace, Int J Prod Res, 55, 1336-1349 (2017)

9. Z.X.Yang, W. Xu, P.K. Wong, X.B.Wang, Math Probl Eng, 575402 (2015)

10. M.Liukkonen, Int J Comput Integ M, 28, 861-880 (2015)

11. K.M.Chen, J.C.Chen, R.A. Cox, Assembly Autom, 32, 185-196 (2012)

12. H.H. Cheung, S.H. Choi, Comput Ind, 62, 708-718 (2011)

13. N.Fescioglu-Unver, S.H. Choi, D. Sheen, S. Kumara, Inform Syst Front, 17, 1369-1380 (2016)

14. H.A.Ringsberg, V. Mirzabeiki, Brit Food J, 116, 104-124 (2014)

15. M. Keskilammi, L. Sydanheimo, M. Kivikoski, Int J Adv Manuf Tech, 21, 769-774 (2003)

16. G.Huang, J.H. Li, X.D. Yuan, L.Gao, Y.Q. Rao, Int J Comput Integ M, 25, 66-85 (2012)

17. E.W.T. Ngai, D.C.K. Chau, J.K.L. Poon, A.Y.M. Chan, J Eng Tech M, 29, 112-130 (2012)

18. P.J.Zelbst, K.W.Green, V.E.Sower, P.M. Reyes, Int. J. Oper. \& Prod. Manage., 32, 329-350 (2012)

19. J.R. Hylsberg, Q. Zhang, T.S. Toftegaard, C. Turcu, (ed.)., InTech 18, 319-334 (2011)

20. Maćkowiak, K. Sieczkarek, M. Logistyka, 4, 46334639 (2014)

21. M. Łobaziewicz, A. Maćkowiak, K. Sieczkarek, Logistyka, 3, 39-42 (2014)

22. Patent PL 222069 B1, Polish Patent Office, (2016)

23. A.Maćkowiak, K. Sieczkarek, Logistyka, 1, 41 (2013)

24. DataConsult $\mathrm{Sp}$. $\mathrm{Z}$ o.o., Communication model between two measuring points in a production system, Research report, 1-16 (2014) 